\title{
EFFECT OF STIMULATED AND THERMAL DESORPTION IN DARHT-2
}

\author{
T. P. Hughes*, MRC, Albuquerque, NM, USA, H. Davis ${ }^{\dagger}$ LANL, Los Alamos, NM, USA
}

\begin{abstract}
The DARHT-2 accelerator generates a $2 \mathrm{kA}, 18 \mathrm{MeV}$, $2 \mu$ sec flat-top electron beam. The beam risetime is about $700 \mathrm{~ns}$, and a "beam cleanup zone" (BCUZ) has been designed to scrape off these mismatched electrons. Experiments on DARHT-1 (which has a 60 ns flat-top) have provided excellent quantitative data on stimulated and thermal desorption of neutral monolayers on various metal surfaces by multi-MeV electrons. We have used these data in the particle-in-cell code LSP to model the production of ions from the walls of the DARHT-2 BCUZ. The effect of these ions on the transport of the main beam pulse is discussed.
\end{abstract}

\section{INTRODUCTION}

The DARHT-2 linear induction accelerator [1] is designed to produce a $2 \mathrm{kA}, 18 \mathrm{MV}, 2 \mu$ s flat-top electron beam. The injector is driven directly by a Marx bank, and has a long voltage risetime: $1-99 \%$ in $700 \mathrm{~ns}$. As a result, there is a considerable amount of beam charge which is mismatched to the solenoid transport channel. The design of a "beam cleanup zone" (BCUZ) to filter out this charge was previously described [2]. In this paper, we present a computational estimate of the ion charge produced by beam electrons striking the walls of the BCUZ. The computational model uses data from experiments carried out on DARHT-1 [3], a companion accelerator with a $60 \mathrm{~ns}$ beam pulse [1].

\section{COMPUTATIONAL MODEL}

\section{Beam Generation}

The DARHT-2 injector geometry is shown in Fig. 1. A $10 \mathrm{~m}$ sections of beam pipe is modeled in $2 \frac{1}{2}$-D using the electromagnetic particle-in-cell (PIC) simulation code LSP[4]. The transmission line attached to the radial boundary at $T_{A K}$ in Fig. 1 produces the voltage pulse shown in Fig. 2 [5]. The cathode is treated as a zero-work-function, space-charge-limited emitter. Emitted electrons are given a transverse temperature corresponding to the surface temperature $\left(\approx 1000^{\circ} \mathrm{C}\right)$. The beam electrons pass through the accelerating gaps and solenoidal fields of the first eight accelerating cells. As in the physical accelerator, each gap in the simulation is powered by a separate transmission line attached at the boundary $\left(T_{1}-T_{8}\right.$ in Fig. 1$)$. The accelerating voltage, also shown in Fig. 2, is based on the experimentally-measured voltage trace [5]. The magnetic tune, shown in Fig. 3 was chosen to avoid any beam-loss

\footnotetext{
*tph@mrcabq.com

$\dagger$ davis@lanl.gov
}

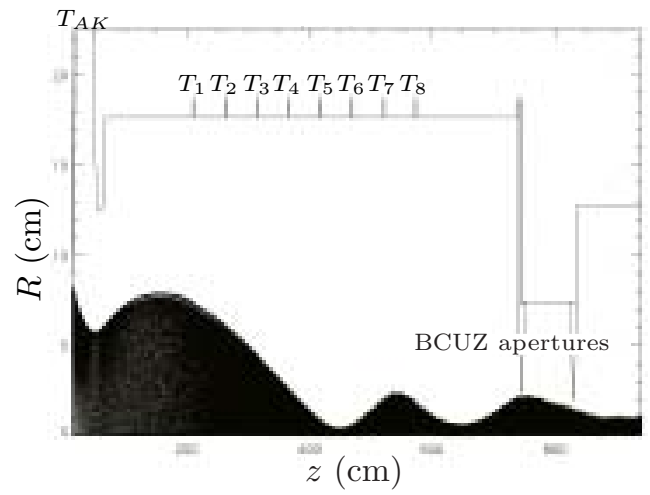

Figure 1: Geometry of desorption calculation, showing injector, 8 accelerating gaps, and BCUZ. The beam is shown at the flat-top energy and current.

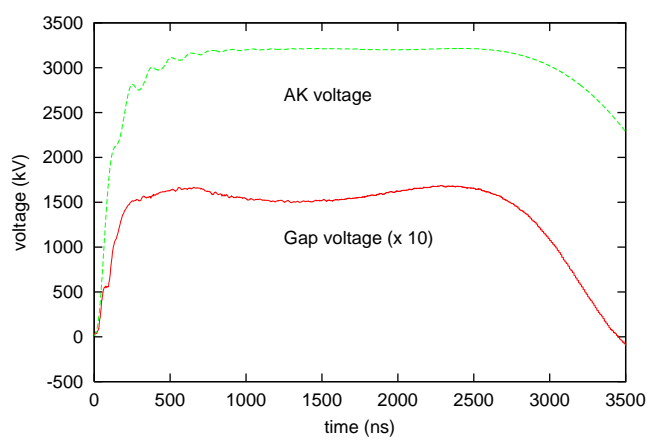

Figure 2: Voltage pulse applied to AK gap (green) and to accelelerating gaps (red). The latter has been multiplied by 10 for scaling purposes.

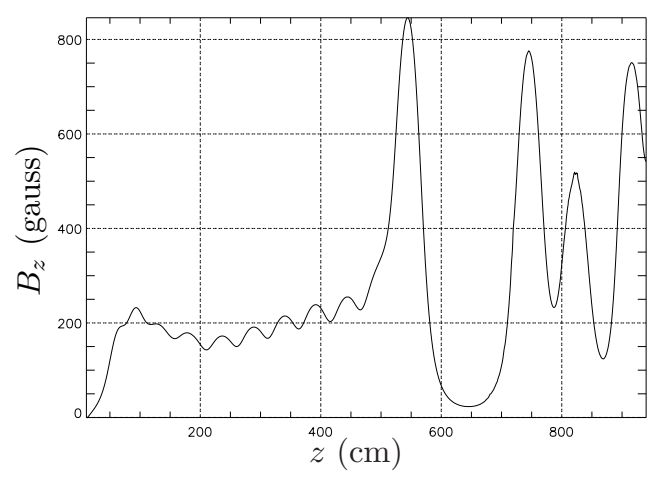

Figure 3: Axial magnetic field tune used in Fig. 1. 
in the accelerating cells, and to scrape off the beam-head in the BCUZ[2]. In the simulation, we see no beam loss until the start of the BCUZ, about 6 meters from the cathode.

\section{Ion Generation Model}

When energetic electrons strike a solid surface, they can generate neutral molecules and ions. There are two mechanisms for generating neutrals: stimulated desorption (ESD) and thermal desorption. Ions can be generated directly by ESD and by a two-step process of neutral desorption followed by ionization. In LSP, these processes are modeled by the following equations:

$$
\begin{aligned}
\frac{d N_{d}^{n}}{d t} & =\frac{j_{e}}{e} \sigma_{d}^{n} N_{a}+N_{a} \nu \exp \left(-\frac{Q_{b}}{T}\right) \\
\frac{d N_{d}^{+}}{d t} & =\frac{j_{e}}{e} \sigma_{d}^{+} N_{a} \\
\frac{d\left(N_{d}^{n}+N_{d}^{+}\right)}{d t} & =-\frac{d N_{a}}{d t} \\
\frac{d N_{i}}{d t} & =\frac{j_{e}}{e} \sigma_{i} N_{d}
\end{aligned}
$$

where $N_{d}^{n}$ is the area density of desorbed neutral particles, $N_{a}$ is the area density of adsorbed particles, $N_{d}^{+}$is the area density of desorbed ions, $N_{i}$ is the area density of ions due to gas-phase ionization, $\sigma_{d}^{n}, \sigma_{d}^{+}$and $\sigma_{i}$ are the crosssections for stimulated desorption of neutral species, stimulated desorption of ionized species, and gas-phase ionization of the neutral species, respectively, $j_{e}$ is the electron current density striking the wall, $\nu$ is a thermal-desorption rate-constant (typically $10^{13} \mathrm{~s}^{-1}$ ), $Q_{b}$ is the binding energy of the adsorbed material in $\mathrm{eV}$, and $T$ is the surface temperature in $\mathrm{eV}$.

In the calculation, we initialize the surfaces with one monolayer $\left(10^{15} \mathrm{~cm}^{-2}\right)$ of neutral water. In the DARHT1 experiments [3], the stimulated neutral desorption yield, $N_{a} \sigma_{d}^{n}$, was measured to be in the range $0.1-0.2$, and the adsorbed inventory was estimated to be about 1 monolayer, mainly consisting of water. Thermal desorption became significant when the surface temperature increased by 300 $400^{\circ} \mathrm{C}$. Roughly, a desorption rate of one monolayer/ns occurs when the surface temperature reaches $Q_{b} / 9 \mathrm{eV}$, which corresponds to about $630 \mathrm{~K}\left(\approx 330^{\circ} \mathrm{C}\right.$ above room temperature) for $Q_{b}=0.5 \mathrm{eV}$, a typical value for water vapor [6]. At room temperature $(300 \mathrm{~K})$ the desorption rate is a factor of $2 \times 10^{4}$ smaller.

Neutrals produced by either stimulated or thermal desorption can be ionized by subsequent beam electrons. We use the gas-phase cross-section for water molecule ionization by relativistic electrons: $\sigma_{i}=0.9 \times 10^{-18} \mathrm{~cm}^{2}$ [7]. Water is known to "crack" under electron impact, producing significant fractions of $\mathrm{OH}^{+}$and $\mathrm{H}^{+}$, in addition to $\mathrm{H}_{2} \mathrm{O}^{+}$[8]. We have not included these species in the calculation.

Direct stimulated production of ions was not measured in the DARHT- 1 experiments. Typically, the cross-section for producing ions is much less than that for neutrals [9].

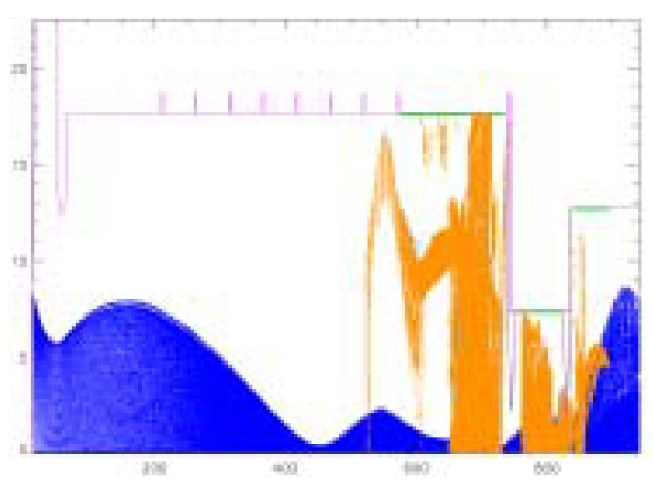

Figure 4: Beam (blue) and $\mathrm{H}_{2} \mathrm{O}^{+}$(orange) distributions at $t=1000$ ns; cf. Fig. 1 .

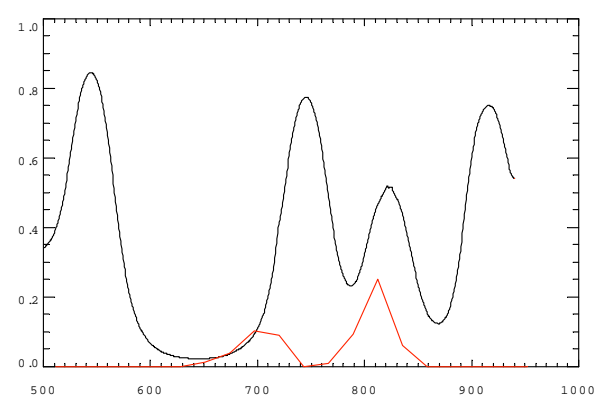

Figure 5: Ratio of ion $\left(\mathrm{H}_{2} \mathrm{O}^{+}\right)$charge to beam charge vs. $z$ at $t=1000 \mathrm{~ns}$, within a radius of $1.5 \mathrm{~cm}$ (red line). The axial solenoidal magnetic field in kilogauss is overplotted (black). Horizontal scale is in $\mathrm{cm}$.

We have used a value $\sigma_{d}^{+}=0.01 \sigma_{d}^{n}=2 \times 10^{-18} \mathrm{~cm}^{2}$ in the calculation.

\section{EFFECT ON THE ELECTRON BEAM}

Ions resulting from stimulated desorption or from ionization of desorbed neutrals can affect the tune and stability of the electron beam. A snapshot of the particle distribution at $t=1000 \mathrm{~ns}$ is shown in Fig. 4. By this time, about $1000 \mu \mathrm{C}$ of beam electron charge has struck the wall, yielding about $2 \mu \mathrm{C}$ of stimulated ion charge. Taking the line-ratio of stimulated ion charge to beam charge within a radius of $1.5 \mathrm{~cm}$ from the axis, we get the results shown by the red line in Fig. 5. The dominant contribution to the ion line-density is from stimulated ions. The number of ions generated from desorbed neutrals is much less: the surface temperature rises by at most $50^{\circ} \mathrm{C}$, as shown in Fig.6. From Fig. 7 is clear that the ions have a large effect on the beam exiting the BCUZ. We can convert the linecharge ratio $f$ to an equivalent magnetic field through the relation

$$
B_{\text {eff }} \approx 3.4 \sqrt{2 \nu \gamma f} / r_{b} \quad \mathrm{kG}
$$




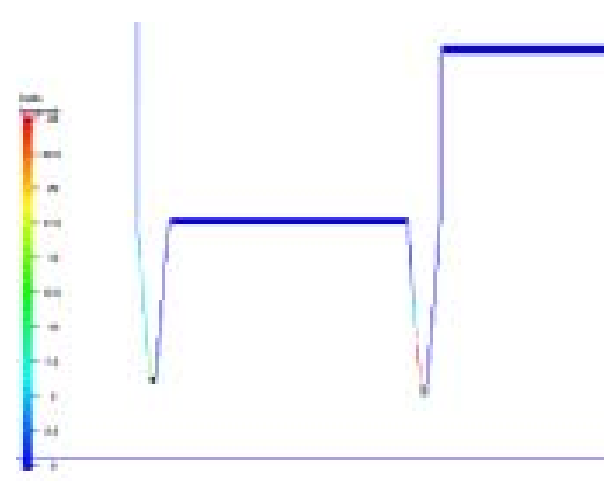

Figure 6: Surface temperature rise (K), in the BCUZ region at the end of the beam risetime.

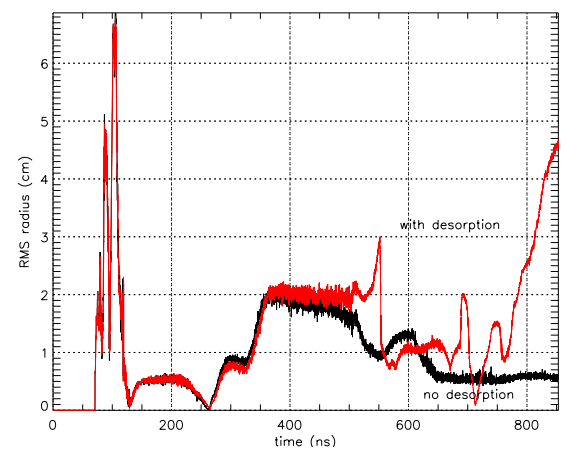

Figure 7: RMS beam radius at $z=938 \mathrm{~cm}$ with (red line) and without (black line) the effect of desorbed ions.

where $\nu$ is Budker's parameter for the beam current and $r_{b}$ is the beam radius in $\mathrm{cm}$. Thus, the first peak in $f$ in Fig. 5 is roughly equivalent to a $1 \mathrm{kG}$ field extending over $30-40 \mathrm{~cm}$, comparable to the actual focusing solenoids in Fig. 5.

\section{CONCLUSIONS}

Beam deposition on the walls of the DARHT-2 beam cleanup zone generates ions through direct stimulated desorption and through neutral desorption followed by impact ionization. For lack of data, the stimulated ion yield used in the calculation is a free parameter. For a sample value equal to $1 \%$ of the measured neutral yield, there is a large disruption of the beam. Experimental data on the stimulated ion yield is needed to make a prediction for how large the effect will be in the actual machine.

\section{ACKNOWLEDGMENTS}

This work was supported by Los Alamos National Laboratory. The simulations were carried out on the Qsc parallel computer under an Institutional Computing grant from LANL.

\section{REFERENCES}

[1] M.J. Burns, B.E. Carlsten, H.A. Davis, C.A. Ekdahl, C.M. Fortgang, B.T. McCuistian, F.E. Merrill, K.E. Nielsen, C.A. Wilkenson, K.P. Chow, W.M. Fawley, H.L. Rutkowski, W.L. Waldron, S.S. Yu, G.J. Caporaso, Y.-J. Chen, E.G. Cook, S. Sampayan, J.A. Watson, G.A. Westenskow, and T.P. Hughes. Status of the DARHT phase 2 long-pulse accelerator. In Proceedings of the 2001 Particle Accelerator Conference, number 01CH37268C (http://accelConferenceweb.cern.ch/AccelConf/p01/PAPERS/WOAA008.PDF).

[2] T.P. Hughes, D.P. Prono, W.M. Tuzel, and J.R. Vananne. Design of beam cleanup zone for DARHT-2. In Proceedings of the 2001 Particle Accelerator Conference, number $01 \mathrm{CH} 37268 \mathrm{C}$, (http://accelConferenceweb.cern.ch/AccelConf/p01/PAPERS/RPPH038.PDF).

[3] H. Davis, D. Moir, and R. Olson. Measurements of thermally desorbed ions from beam-target interactions. In Proceedings of the 2003 Particle Accelerator Conference, 1993. Paper ROPB003.

[4] LSP is a software product of Mission Research Corporation (http://www.mrcabq.com).

[5] M. Kang and K. Nielsen, 2003. Private communication.

[6] M.E. Cuneo. The effect of electrode contamination, cleaning and conditioning on high-energy pulsedpower device performance. IEEE Trans. Dielectr. Electr. Insul., 6:469, 1999.

[7] T. C. Genoni and T. P. Hughes. Ion-hose instability in a long-pulse linear induction accelerator. Phys. Rev. ST Accel. Beams, 6, 030401, 2003.

[8] M. V. V. S. Rao, I. Iga, and S.K. Srivastava. Ionization cross-sections for the production of positive ions from H2O by electron impact. J. Geophys. Res., 100:26421, 1995.

[9] T. E. Madey and J. T. Yates, Jr. Electron-stimulated desorption as a tool for studies of chemisorption: A Review. J. Vac. Sci. Technol., 8(4):525, 1971. 